\title{
A new equation of state Based on Nuclear Statistical Equilibrium for Core-Collapse Simulations
}

\author{
Shun Furusawa ${ }^{1}$, Shoichi Yamada ${ }^{1,2}$, Kohsuke Sumiyoshi ${ }^{3}$ and \\ Hideyuki Suzuki ${ }^{4}$ \\ ${ }^{1}$ Department of Science and Engineering, Waseda University, 3-4-1 Okubo, Shinjuku, Tokyo \\ 169-8555, Japan \\ email: furusawa@heap.phys .waseda.ac.jp \\ ${ }^{2}$ Advanced Research Institute for Science and Engineering, Waseda University, 3-4-1 Okubo, \\ Shinjuku, Tokyo 169-8555, Japan \\ ${ }^{3}$ Numazu College of Technology, Ooka 3600, Numazu, Shizuoka 410-8501, Japan \\ ${ }^{4}$ Faculty of Science and Technology, Tokyo University of Science, Yamazaki 2641, Noda, Chiba \\ 278-8510, Japan
}

\begin{abstract}
We calculate a new equation of state for baryons at sub-nuclear densities for the use in core-collapse simulations of massive stars. The formulation is the nuclear statistical equilibrium description and the liquid drop approximation of nuclei. The model free energy to minimize is calculated by relativistic mean field theory for nucleons and the mass formula for nuclei with atomic number up to $\sim 1000$. We have also taken into account the pasta phase. We find that the free energy and other thermodynamical quantities are not very different from those given in the standard EOSs that adopt the single nucleus approximation. On the other hand, the average mass is systematically different, which may have an important effect on the rates of electron captures and coherent neutrino scatterings on nuclei in supernova cores.
\end{abstract}

Keywords. equation of state, dense matter, nuclear abundances \& supernovae

Equations of state (EOSs) of hot and dense matter play an important role in the dynamics of core-collapse supernovae both at sub- and supra-nuclear densities. In addition to thermodynamical quantities such as pressure, information on matter composition is provided by EOS. At sub-nuclear densities, the latter affects the rates of electron captures and coherent neutrino scatterings on nuclei, both of which in turn determine the electron fraction of collapsing cores, one of the most critical ingredients for the core dynamics.

At present, there are only two EOSs in wide use for the core-collapse simulation. Lattimer et al. (1991)'s EOS is based on Skyrme-type nuclear interactions and the so called compressible liquid drop model for nuclei surrounded by dripped nucleons. The EOS by H. Shen et al. (1998) et al. employs a relativistic mean field theory (RMF) to describe nuclear matter and the Thomas Fermi approximation for finite nuclei with dripped nucleons. It should be emphasized here that both EOSs adopt the so-called single nucleus approximation (SNA), in which only a single representative nucleus is included. In other words, the distribution of nuclei is ignored. There are a few EOSs assuming multinuclei (Botvina et al. (2010), Hempel et al. (2010) \& G. Shen et al. (2011)). However, there is no model that takes into account both shell effects of nuclei at low densities and nuclear pasta phase transition to uniform matter with multi-nuclei at high densities.

The aim of our project is in a sense to merge all the previous EOSs to provide all the information needed for core-collapse simulations: thermodynamical quantities such as pressure as well as the information on the abundance of nuclei required for the calculation 


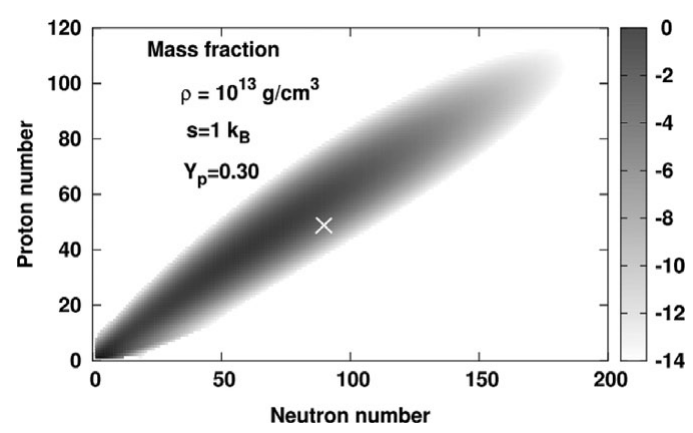

Figure 1. The mass fractions of nuclei in the $(N, Z)$ plane for $\rho_{B}=10^{11} \mathrm{~g} / \mathrm{cm}^{3}, T=1 \mathrm{MeV}$ and $Y_{p}=0.3$. The cross indicates the representative nucleus for the H. Shen's EOS under the same condition.

of the weak interaction rates. The description of uniform nuclear matter is based on RMF in H. Shen's EOS and the approximate treatment of the pasta phase is similar to the one employed in Lattimer-Swesty's EOS. We solve a Saha-like equation to obtain the abundance of nuclei.

The formulation of this model is based on the NSE description using the mass descriptions for nuclei up to the atomic number of 1000 under the influence of surrounding nucleons and electrons. The mass data are taken from the experimental nuclear mass that allow us to take into account the nuclear shell effects. An extended liquid drop model is used to describe the nuclear mass whenever the experimental data are not available together with the medium effects, and in particular, the formulation of the pasta phases. Because of this combination of the mass data and the theoretical model, the free energy of multi-component system can reproduce the ordinary NSE results at low densities and make a continuous transition to the EOS for supra-nuclear densities. The details are given in Furusawa et al. (2011).

We find that the thermodynamical quantities such as free energy and pressure per baryon agree well among other EOSs. The matter compositions show noticeable differences, however. The average mass numbers, for example, are systematically smaller in our EOS than the mass numbers of the representative nuclei in the H.Shen EOS. We can see that the representative nucleus of H.Shen EOS is different from the abundance peak of our model in Fig.1. In other words, SNA can not reproduce the ensemble of nuclei.

We are currently constructing an EOS table, which will be available for supernova simulations and made available in the public domain. In so doing, electron capture rates, which are consistent with the abundance given by the EOS, should also be included.

\section{References}

Furusawa, S., Yamada, S., Sumiyoshi, K., \& Suzuki, H. 2011, ApJ, 738, 178

Shen, G., Horowitz, C. J., \& Teige, S. 2011, Phys. Rev. C, 83, 035802

Shen, H., Toki, H., Oyamatsu, K., \& Sumiyoshi, K. 1998, Nucl. Phys., A637, 435

Lattimer, J. M. \& Swesty, F. D. 1991, Nucl. Phys., A535, 331

Botvina, A. S. \& Mishustin, I. N. 2010, Nucl. Phys. A 843, 98

Hempel, M. \& Schaffner-Bielich, J. 2010, Nucl. Phys. A 837, 210 